21 Manuscript: Pages - 52; Tables - 2; Figures - 8; Supplemental Figures - 2

\section{Retinal and Cortical Determinants of Cortical Magnification in Human Albinism}

\author{
Erica N. Woertz, ${ }^{a}$ Melissa A. Wilk, ${ }^{a, *}$ Ethan Duwell, ${ }^{b}$ Jedidiah Mathis ${ }^{b, c}$, Joseph
}

$$
\text { Carrolla,b,d, Edgar A. DeYoe }{ }^{b, c, * *}
$$

a Department of Cell Biology, Neurobiology, \& Anatomy, Medical College of Wisconsin, 8701 Watertown Plank Road, Milwaukee, WI 53226, USA

${ }^{\mathrm{b}}$ Department of Biophysics, Medical College of Wisconsin, 8701 Watertown Plank

(1)

Road, Milwaukee, WI 53226, USA

c Department of Radiology, Medical College of Wisconsin, 8701 Watertown Plank Road, Milwaukee, WI 53226, USA

d Department of Ophthalmology \& Visual Sciences, Medical College of Wisconsin, 925 N. $87^{\text {th }}$ Street, Milwaukee, WI 53226, USA

*Current affiliation: HudsonAlpha Institute for Biotechnology, 601 Genome Way, NW, Huntsville, AL 35806 ${ }^{* *}$ Corresponding Author: Edgar DeYoe, Department of Radiology, Medical College of Wisconsin, 8701 Watertown Plank Road, Milwaukee, WI 53226; deyoe@mcw.edu

23 CONFLICTS OF INTEREST: The authors declare no competing financial interests. 
25 ACKNOWLEDGEMENTS:

26 The authors would like to thank Brittany Bartlein, Robert Cooper, and Phyllis Summerfelt

27 for their contributions to this work. Research reported in this publication was supported

28 by the National Eye Institute, the National Institute of General Medical Sciences, and the

29 National Center for Advancing Translational Sciences of the National Institutes of Health

30 under award numbers TL1TR001437, T32GM080202, T32EY014537, P30EY001931,

31 and R01EY024969. This investigation was conducted in a facility constructed with support

32 from Research Facilities Improvement Program, Grant Number C06RR016511, from the

33 National Center for Research Resources, National Institutes of Health. The content is

34 solely the responsibility of the authors and does not necessarily represent the official

35 views of the National Institutes of Health. This work was also supported by Vision for

36 Tomorrow and the Thomas M. Aaberg, Sr., Retina Research Fund. 
ABSTRACT

The human fovea lies at the center of the retina and supports high-acuity vision. In normal visual system development, foveal acuity is correlated with both a high density of

41 cone photoreceptors at this location and a magnified retinotopic representation of the

42 fovea in the visual cortex. Both cone density and the cortical area dedicated to each

43 degree of visual space-the latter known as the cortical magnification function-steadily

44 decline with increasing eccentricity from the fovea. In albinism, peak cone density at the

45 fovea and visual acuity are reduced but appear to be normal in the periphery, thus

46 providing a model to explore the correlation between retinal structure, cortical structure,

47 and behavior. Here, we used adaptive optics scanning light ophthalmoscopy to assess

48 retinal cone density and functional magnetic resonance imaging to measure cortical

49 magnification in primary visual cortex of normal controls and individuals with albinism. We

50 find that retinotopic organization is more varied in albinism than previously appreciated,

51 yet cortical magnification outside the fovea is similar to that in controls. Moreover, cortical

52 magnification in albinism and controls exceeds that which might be predicted based on

53 cone density alone, suggesting that reduced foveal cone density in the albinotic retina

54 may be partially counteracted by central connectivity. Together, these results emphasize

55 that central as well as retinal factors must be included to provide a complete picture of 56 aberrant structure and function in genetic conditions such as albinism. 


\section{INTRODUCTION}

The human fovea occupies about $0.02 \%$ of the total retinal area, but is responsible for our highest-acuity vision, with approximately $40 \%$ of primary visual cortex (V1)

61 dedicated to processing its signals (Hendrickson, 2005). The fovea is characterized by

62 the excavation of inner retina, a lack of retinal vasculature, and a small region absent of

63 rod photoreceptors. The fovea has the highest density of cone photoreceptors, as well as

64 non-convergent connections between these cones and their post-synaptic partners,

65 known as the "midget" system (Curcio and Allen, 1990; Dacey, 1993). This leads to

66 cortical sampling that is up to 160 times greater than that of peripheral cones (Duncan

67 and Boynton, 2003). Cone density declines with increasing eccentricity, with the steepest

68 decline occurring within 1-2 $\mathrm{mm}$ of the fovea (Curcio et al., 1990). Likewise, in V1 the

69 amount of cortical space devoted to each degree of visual angle is grossly magnified at

70 the fovea and decreases steadily with increasing eccentricity (Daniel and Whitteridge,

71 1961; Cowey and Rolls, 1974).

Behaviorally, this relationship between cortical magnification (CM) and eccentricity

73 is directly correlated with visual acuity. This was shown with the earliest empirical

74 measurements of CM in humans using electrodes implanted in the occipital lobe (Cowey

75 and Rolls, 1974). Later studies using functional magnetic resonance imaging (fMRI)

76 showed strikingly similar CM measurements, though there is some minor variability that

77 has been attributed to disparities in stimulus design and methods used to measure

78 cortical distance (Sereno et al., 1995; Engel et al., 1997; Popovic and Sjöstrand, 2001;

79 Duncan and Boynton, 2003; Qiu et al., 2006). 
While methodological differences may contribute to this variation in empirical measurement of $\mathrm{CM}$, it is also possible that some variation comes from actual anatomical differences between individuals. In the retina, histologic studies show more than a 3-fold

83 difference in foveal cone density across individuals (Curcio et al., 1990), and the sizes of

84 the optic tract, lateral geniculate nucleus (LGN), and V1 are known to be correlated within

85 individuals (Andrews et al., 1997). Since foveal magnification in the visual cortex is driven in part by the relatively higher cone density found at the fovea, it follows that CM should vary across subjects as a function of cone density (Dougherty et al., 2003). Recent advances in retinal imaging with adaptive optics has made it possible to resolve the cone mosaic in the living human eye, providing the opportunity to assess cone density and CM

90 in the same individual. Such comparisons can determine whether variation in retinal 91 structure correlates with variation in CM. Extreme examples of individual variation are found certain pathologies. One 93 notable example is albinism, a family of genetic diseases that disrupt melanin synthesis 94 and cellular trafficking, resulting in abnormal development of the visual system 95 (Nettleship, 1909; Creel et al., 1978; O'Donnell et al., 1978). This leads to greater visual system variability among individuals with albinism than among normal individuals, with

97 peak cone density that is (on average) lower than that observed in normal individuals 98 (Wilk et al., 2014). Thus, albinism is an advantageous model to probe structural correlates 99 of variability in CM. Additionally, individuals with albinism are known to experience 100 decreased best-corrected visual acuity compared to normal individuals (Wilson et al., 101 1988; Summers, 1996). Although they generally have foveal cone densities below the 102 normal range of $100,000-320,000$ cones $/ \mathrm{mm}^{2}$ (Curcio et al., 1990) with minimum foveal 
103 cone densities as low as 29,000 cones $/ \mathrm{mm}^{2}$, their visual acuity and cone density does

104 not appear to be precisely correlated (Wilk et al., 2014). Thus, the physiological source

105 of their acuity deficits remains unclear. Several studies have assessed cortical

106 reorganization in albinism (Morland et al., 2001; Hoffmann et al., 2003; von dem Hagen

107 et al., 2005; von dem Hagen et al., 2007; Neveu et al., 2008; von dem Hagen et al., 2008;

108 Bridge et al., 2014; Ather et al., 2019), but these studies lacked molecular genotyping to

109 confirm the specific subtypes of albinism included in their cohorts. Moreover, to our

110 knowledge no studies in this population have assessed CM and its quantitative

111 relationship to cone density. In light of the above-mentioned relationship between visual

112 acuity and $\mathrm{CM}$ in normal individuals, it is of particular interest to determine how CM in

113 albinism compares to that in normal individuals and if it correlates with observed

114 decreases in cone density at different eccentricities. This will provide a bridge between

115 visual system structure and aberrant acuity functions in albinism, as well as insight into

116 the nature of pathological retino-cortical relationships in disease.

Here, we performed high-resolution retinal imaging using adaptive optics scanning

118 light ophthalmoscopy (AOSLO) and retinotopic mapping in the visual cortex using fMRI

119 to examine both cone density and $\mathrm{CM}$ in the normal and albinotic visual system. We

120 hypothesized that CM would be reduced in subjects with albinism in proportion to their

121 unique pattern of reduced cone density versus visual field eccentricity. However, our

122 results suggest that cone density is not the sole determinant of CM and its variability.

123 Additional central factors are required to account for the aberrant cortical patterns within

124 this population. 
METHODS

127 Subjects

128 Six subjects with albinism (4 females, 2 males; aged 15-31 years) with minimal 129 nystagmus and five subjects with no prior ocular or cortical pathology (2 females, 3 males; 130 aged 20-25 years) were recruited for this experiment. One subject with albinism was 131 excluded from further analysis due to significant motion artifacts in the fMRI (male, age 13215 years); subjects included in the analysis are listed in Table 1. Retinal features of these 133 subjects have been previously described (Wilk et al., 2014; Wilk et al., 2017). The study 134 was in accordance with the Declaration of Helsinki and approved by the Institutional 135 Review Board of the Medical College of Wisconsin. All subjects provided written consent 136 after explanation of the nature and possible consequences of the study.

138 Table 1. Subject demographics.

\begin{tabular}{ccccccc}
\hline Condition & Subject & Sex & Age & Eye & $\begin{array}{c}\text { Foveal } \\
\text { (mm) }\end{array}$ & $\begin{array}{c}\text { Axial Length } \\
\text { Cone Density } \\
(\text { cones/mm }\end{array}$ \\
\hline \multirow{4}{*}{ Normal }
\end{tabular}

$139{ }^{*}$ Cone density for this subject was previously reported by Wilk et al. (2014) 
${ }^{* *}$ Cone density for this subject was previously reported by Wilk et al. (2017)

Fixation Testing

Fixational stability was assessed in all subjects with albinism using the fixation test module on the OPKO combined scanning laser ophthalmoscope (SLO) and optical

145 coherence tomography (OCT) imaging system. After the SLO scanner was focused on 146 the subject's retina, the operator specified a group of inner retinal blood vessels to track 147 for the duration of the run. The subject was instructed to fixate a small white cross during 148 the run while minimizing blinks. Subjects completed three 20 -second runs. Subsequently, the reference SLO images used in each run were manually registered to each other using 150 only translation and rotation in Adobe Photoshop CS6 (Adobe Systems, Inc., San Jose, 151 CA). The transform for each SLO image was then applied to the corresponding fixation 152 coordinates, and the fixation points from all runs were combined to calculate the $68 \%$ and 153 95\% bivariate contour ellipse area (BCEA) using the following equations:

(1) $B C E A_{50}=1.38 \pi \sigma_{H} \sigma_{V}\left(1-\rho^{2}\right)^{\frac{1}{2}}$

(2) $B C E A_{95}=6.00 \pi \sigma_{H} \sigma_{V}\left(1-\rho^{2}\right)^{\frac{1}{2}}$

Where $\sigma_{\mathrm{H}}$ and $\sigma_{\mathrm{V}}$ are the standard deviations of the coordinates in the horizontal and 157 vertical directions (respectively) and $\rho$ is product-moment correlation of the horizontal and vertical coordinates (Steinman, 1965; Crossland et al., 2004).

\section{Retinal Imaging}

Axial length of the eye (used for lateral scaling of retinal images) was obtained for 162 each subject using an IOL Master (Carl Zeiss Meditec, Dublin, CA). One eye for each 
163 subject was dilated and accommodation was suspended using one drop each of

164 Phenylephrine Hydrochloride (2.5\%) and Tropicamide (1\%). A previously described AOSLO (Dubra and Sulai, 2011) was used to obtain images of the photoreceptor mosaic 166 at the fovea and a strip in the temporal retina. To produce an image with minimal 167 distortion, raw videos were first "desinusoided" to correct for the sinusoidal motion of the 168 resonant scanner by estimating the distortion from images of a Ronchi ruling and then 169 resampling the images over a grid of equally spaced pixels (Cooper et al., 2011). The 170 videos were then manually inspected for reference frames that contained minimal 171 distortion, which were then used for image registration using custom software (Dubra and 172 Harvey, 2010). Registered images were manually aligned using Adobe Photoshop 173 (Adobe Systems, Inc., San Jose, CA). Foveal cones were identified using a previously 174 described, semi-automated algorithm (Garrioch et al., 2012). The location of peak cone 175 density was identified and cone density was measured at locations across the temporal 176 retina using a $37 \mu \mathrm{m}$ sampling window as previously described (Wilk et al., 2014). For 177 images within approximately $2^{\circ}$ of the fovea, cone counting was done using the same 178 semi-automated algorithm (Garrioch et al., 2012). For peripheral images, cones were 179 identified manually by a single observer (MAW) using ImageJ (Schneider et al., 2012) or 180 custom Java (Oracle Corporation, Redwood Shores, CA) software (Cooper et al., 2016).

fMRI Visual Stimuli

All fMRI stimuli were presented on a back-projection screen mounted on the MR 184 head coil using a BrainLogics BLMRDP-A05 MR digital projector (Puckett and DeYoe, 
2015). Stimuli were generated using a ViSaGe MKII visual stimulus generator (Cambridge

Research) in conjunction with MATLAB.

Stimuli included conventional expanding ring and rotating wedge retinotopic mapping stimuli (DeYoe et al., 1996). Rings and wedges were composed of black and white counterphase flickering circular checkerboards $(8 \mathrm{~Hz})$ with check size and ring width scaled with eccentricity. Stimuli were presented on a uniform gray background and subtended a maximum of $20^{\circ}$ eccentricity. All subjects were instructed to continually fixate at the center of the screen. To enhance stable fixation, thin, black radial lines extending from fixation to the edge of the display were present continuously in all tasks.

To minimize unnecessary duplication, retinotopic mapping data for normal control subjects were obtained using a different experimental protocol than for the subjects with albinism. Consequently, there were slight differences in some stimulus parameters. 197 However, due to the temporal phase mapping methods used in this study these differences did not significantly affect our results. For subjects with albinism, the wedge stimuli subtended $45^{\circ}$ polar angle, and for control subjects the wedges subtended $90^{\circ}$.

200 All subjects viewed both ring and wedge stimuli binocularly with full-field stimulation. Additionally, for subjects with albinism the expanding ring stimuli were presented to the 202 right and left hemifields in separate runs and were tested separately for each eye. The hemifield ring stimuli were identical to the full field version, except that one hemifield was

204 masked to match the grey background. For control subjects, the ring stimulus expanded 205 from the center to the periphery in 40 seconds and was repeated five times per run. For 206 subjects with albinism, both the full-field and hemifield ring stimuli expanded from $0.8^{\circ}$ 207 eccentricity to the periphery in 60 seconds and was repeated five times per run. For 
subjects with albinism, the center of the display consisted of a circular black and white disc (similar to a radioactivity symbol) with a radius of $0.8^{\circ}$ that flickered at random intervals not synchronized to the rings/wedges presentation. To control attention, subjects were instructed to press and hold a button whenever the pattern appeared.

\section{fMRI Stimulus Paradigm} tasks in the first session, and all remaining tasks in the second session. All monocular

218 times. For monocular stimuli, repetitions of the right and left hemifield stimuli were 219 interleaved; for full-field stimuli, repetitions of the expanding ring and rotating wedge were 220 interleaved. After each fMRI run, the subject was asked to rate their alertness on a scale 221 from 1-5 (1 being asleep and 5 being fully awake). This measure was intended to control 222 for subjects' alertness, which can affect the quality of data and potentially be used as an exclusion criterion. No data were excluded from this study based on the alertness ratings.

\section{fMRI Acquisition} system at the Medical College of Wisconsin. A custom 32-channel RF/Gradient head coil and a $T 2^{*}$-weighted gradient-echo EPI pulse sequence $\left(T E=25 \mathrm{~ms}, T R=2 \mathrm{~s}, \mathrm{FA}=77^{\circ}\right.$ ) 
23129 axial slices in the occipital lobe and adjacent portions of the temporal and parietal

232 lobes with a slice thickness of $2.5 \mathrm{~mm}$, yielding a raw voxel size of $2.5 \mathrm{~mm}^{3}$. The data

233 were Fourier interpolated to $1.875 \times 1.875 \times 2.5 \mathrm{~mm}$. For anatomical scans, a T1-

234 weighted spoiled GRASS pulse sequence was used (SPGR, TE = $3.9 \mathrm{~ms}$, TR = 9.6 ms,

$235 \mathrm{FA}=12^{\circ}$ ) with a $256 \times 224$ acquisition matrix. The FOV was $24 \mathrm{~cm}$, and 220 slices with

236 a slice thickness of $1.0 \mathrm{~mm}$, yielding raw voxel sizes of $0.938 \times 1.07 \times 1.0 \mathrm{~mm}^{3}$. The

237 SPGR scans were Fourier interpolated to $0.938 \times 0.938 \times 1.0 \mathrm{~mm}^{3}$ and subsequently

238 resampled to $1.0 \mathrm{~mm}^{3}$. A sync pulse from the scanner at the beginning of each run

239 triggered the onset of visual stimuli.

241 Analysis Software

All fMRI data were analyzed using the AFNI/SUMA package (Cox, 1996). Surface

243 models were produced from the high-resolution SPGR images using Freesurfer (version

2445.1 .0 or 5.3.0, http://surfer.nmr.mgh.harvard.edu/) with the 'recon-all' function.

fMRI Pre-Processing

fMRI pre-processing was performed in the following order: reconstruction, volume

248 registration, averaging of the time courses, removal of the initial magnetization transients,

249 alignment. Volumes from all individual runs in an interleaved block were registered to the

250 middle volume of the first run in the block using AFNI 3dVolreg. Individual runs for each

251 functional task were averaged using AFNI 3dMean to produce average time courses. The

252 before and after periods were removed using AFNI 3dcalc. 
For subjects with albinism, we attempted to minimize bias in the alignment of

254 functional scans from either of the two sessions to the reference anatomy by using a 255 modified version of the align_across_days.csh script available on the AFNI and NIfTI 256 server (https://sscc.nimh.nih.gov/sscc/dglen/alignmentacross2sessions). In this script,

257 the reference SPGR anatomical images from both sessions were skull-stripped using 258 AFNI 3dSkullStrip, aligned using AFNI align_epi_anat.py script, and averaged using AFNI $2593 d$ Mean to create an average reference anatomy for the two sessions. All individual and 260 average functional runs were then aligned to these average reference anatomies using 261 the align_epi_anat.py script. For control subjects, all data were acquired in a single 262 session, so creation of an average reference anatomy was unnecessary. Functional runs 263 for control subjects were aligned to reference SPGR anatomical scans using the 264 align_epi_anat.py script.

Phase Encoded Retinotopic Maps

Phase encoded retinotopic activation maps were generated to plot the spatial distribution of significant fMRI responses in each of the functional tasks. Significant responses were identified by cross correlating the empirical time course data for each 270 voxel with a reference waveform using AFNI 3ddelay (Bandettini et al., 1993; Saad et al., 271 2003; Datta and DeYoe, 2009). This analysis produces the correlation coefficient and 272 phase values at the phase offset of maximum correlation for each voxel. The reference 273 waveform used for this phase mapping procedure was a binary square wave describing 274 the stimulus cycles convolved temporally with the "Cox Wide" estimation of the 275 hemodynamic response function (HRF). Time courses were spatially smoothed using 
276 AFNI 3dLocalstat with a $3.75 \mathrm{~mm}$ spherical kernel prior to this phase mapping procedure 277 and all functional runs were thresholded to a minimum correlation coefficient of 0.30 .

278 Phase-mapped eccentricity and polar angle values at each voxel were then projected 279 onto functional field maps (FFmaps) using Prism View (Version 4.1.0; Prism Clinical 280 Imaging, Elm Grove, WI) as previously described (Reitsma et al., 2013), and the 281 eccentricity and polar angle FFmaps were manually calibrated (expanded or rotated 282 respectively) so as to accurately represent the corresponding visual stimulus parameter.

283 The correction factor used to make this adjustment, expressed as a constant temporal 284 "offset" (in seconds), was then applied to the temporal delay values for all voxels in 285 subsequent analyses.

\section{V1 Surface Area}

Using polar angle fMRI maps on the inflated cortical surface, V1 boundaries were defined by the superior (ventrally) and inferior (dorsally) vertical meridian representations

290 along the banks of the calcarine sulcus (Figure 1A). A $16^{\circ}$ isoeccentricity boundary from 291 the fMRI eccentricity map of V1 was marked manually on inflated cortical surfaces. This 292 boundary was then connected to the ventral and dorsal boundaries of V1 and a region of 293 interest (ROI) containing all nodes within this region was created. Surface area and 294 cortical volume of V1 within this ROI was calculated using the AFNI SurfMeasures 295 function. Surface area was calculated at both the pial surface and the gray/white matter 296 boundary, then averaged to approximate layer 4 of striate cortex. 
Three linear ROls were drawn on inflated cortical surfaces along and parallel to the horizontal meridian representation within V1 for each subject (Figure 1B). Phaseencoded eccentricity and/or polar angle within the visual field was determined for each surface node using AFNI vol2surf and custom Matlab software. The eccentricity for each node was then matched to its linear distance along each ROI using pial (i.e., non-inflated)

304 surface measurements in AFNI (Figure 1C). The total distance along each ROI was corrected by a factor of 0.93 to account for the small random variations in position of nodes in the cortical surface mesh relative to the ROIs drawn on a smooth inflated surface

307 (correction factor determined by a previous analysis comparing mesh-based ROls with directly-measured distances). Using custom Matlab software, cortical mapping functions were created by plotting the eccentricity represented by each voxel against its corrected distance along the ROI. Although our mapping data extended to $20^{\circ}$, all points beyond

$31116^{\circ}$ were excluded from further analysis, because large population receptive field sizes in

312 the periphery can introduce measurement artifacts in the shape of cortical mapping 313 curves (Baseler et al., 2002). Additionally, in JC_10230 the ROls from one hemisphere in

314 one task were cropped at the beginning of the ROls to eliminate a few highly aberrant 315 measurements that prevented accurate curve fitting. modeled as a function of cortical distance (in millimeters), $d$ : 
322 shown below in equation (4) (Qiu et al., 2006). Using the fminsearch function in Matlab,

323 this curve was first fit to each individual ROI, and then normalized so that $d$ was expressed

324 as the physical distance relative to the location that represented $8^{\circ}$ eccentricity (i.e., when

$325 d=0 \mathrm{~mm}, E(d)=8^{\circ}$ ). A single curve was then fit to all data points from all three ROls. For

326 all subjects, each hemisphere in each task was modeled separately.

The cortical magnification function (CMF) was subsequently computed as the

328 derivative of the inverse of the fitted cortical mapping function, which can be represented 329 analytically (Qiu et al., 2006) as:

(4) $\frac{d d}{d E}=\frac{1}{c * E}$

331 Similarly to the cortical mapping function in equation (3), here we used $1 / c$ as a "CMF 332 scaling factor" to describe the shape of the function. This curve was computed piecewise using custom Matlab software.

For comparison, we proposed a null hypothesis that $\mathrm{CM}$ is solely determined by 335 cone density with no differential convergence or divergence in the connections of those cones at different eccentricities. Accordingly, the cone density for each subject was used

337 to calculate a predicted cortical mapping function based on the assumption that each 338 cone was represented by equal distance in V1. Thus, the mapping of retinal eccentricity to cortical linear distance was computed as:

(5) $d_{\text {cortex }}=\int_{0}^{e c c}$ cone density $(e c c) * \Delta$ dist/cone

341 The inverse of this function provided a predicted cortical mapping function. A predicted

342 CMF was then computed as described above for the empirical cortical mapping functions.

343 The $\Delta$ dist/cone factor was implicitly computed so as to evenly distribute the cumulative 
344 cone count from 0 to 16 degrees over the cortical ROI distance subtending the same 345 eccentricity range.

\section{Statistical Analysis}

All statistical comparisons were performed using Prism 8 (GraphPad Software, 349 Inc.). The Shapiro-Wilk test was used to assess normality, and data were classified as 350 normal when $p>0.05$. Parametric tests (two-tailed $t$-test and paired $t$-test) were used to 351 compare normal data, and non-parametric tests (Mann-Whitney U-test and Wilcoxson 352 matched-pairs test) were used to compare non-normal data. Differences between groups were considered to be significant when $p<0.05$.

\section{RESULTS}

356 Fixational Stability

The $50 \%$ and $95 \%$ BCEA for each eye in each subject with albinism are shown in

Table 2. Subjects had $50 \%$ BCEAs ranging from 0.04 to $5.97 \mathrm{deg}^{2}$ with an average of $3591.40 \mathrm{deg}^{2} .95 \%$ BCEAs ranged from 0.18 to $25.97 \mathrm{deg}^{2}$ with an average of $6.07 \mathrm{deg}^{2}$. Previously, "steady fixation" has been defined as having $50 \%$ of fixation points fall within a $2^{\circ}$-diameter circle (i.e., $3.14 \mathrm{deg}^{2}$ area) centered at the locus of fixation (Fujii et al., 2003). While the BCEA is not necessarily circular, its elliptical shape helps account for

363 the predominantly horizontal eye movements that are typically observed in nystagmus.

364 As indicated by the 50\% BCEAs shown in Table 2, all subjects except for one (JC_10093) 365 had $50 \%$ BCEAs that were less than $3.14 \mathrm{deg}^{2}$. 
367 Table 2: Fixational stability in subjects with albinism

\begin{tabular}{c|cc|cc} 
Subject & \multicolumn{2}{|c|}{ OD } & \multicolumn{2}{c}{ OS } \\
& $50 \%$ BCEA & $95 \%$ BCEA & $50 \%$ BCEA & $95 \%$ BCEA \\
\hline JC_0492 & 0.04 & 0.19 & 0.06 & 0.28 \\
JC_0493 & 0.84 & 3.66 & 0.14 & 0.597 \\
JC_10093 & 3.68 & 16.01 & 5.97 & 25.95 \\
JC_10227 & 0.24 & 1.05 & 0.45 & 1.95 \\
JC_10230 & 1.92 & 8.35 & 0.62 & 2.68
\end{tabular}

$368 \mathrm{OD}=$ right eye; $\mathrm{OS}=$ left eye; $\mathrm{BCEA}=$ bivariate contour ellipse area. All values are

369 expressed in degrees ${ }^{2}$.

370

371 Cone Density

372 Control subjects' foveal peak cone densities ranged from 84,733 to 165,080 373 cones $/ \mathrm{mm}^{2}$ (mean \pm SD: $123,885 \pm 28,760$ cones $/ \mathrm{mm}^{2}$ ), while patients with albinism had

374 foveal cone densities ranging from 46,019 to 89,116 cones $/ \mathrm{mm}^{2}$ (average $\pm \mathrm{SD}=69,248$

$375 \pm 19,623$ cones $/ \mathrm{mm}^{2}$ ). Cone density as a function of eccentricity for each subject can be

376 seen in Figure 2. All subjects showed a reduction in cone density with eccentricity.

377 Patients with albinism had generally lower densities within 3 degrees of the fovea;

378 however, cone density became more similar across all subjects in the periphery (Figure

379 2). Three subjects with albinism (JC_0492, JC_0493, and JC_10227) had peak cone

380 densities that fell within two standard deviations of normal, while the remaining subjects

381 with albinism (JC_10093 and JC_10230) had much lower peak densities. 


\section{Surface Area of V1}

The cortical surface area and volume of $\mathrm{V} 1$ representing the central $16^{\circ}$ of the visual field was measured in all control subjects and four subjects with albinism using manually-drawn ROls based on isopolar and isoeccentricity maps (Figure 3A). V1 was not measured in JC_10230 because the thresholded eccentricity data (i.e., voxels with a correlation coefficient of 0.30 or greater) did not extend all the way to $16^{\circ}$. While surface area appeared to be reduced in albinism relative to controls (Figure 3B), this difference approached but did not achieve statistical significance (two-tailed $t$ test: $t=1.98, \mathrm{df}=16$, $p=0.065)$.

\section{Retinotopy in Albinism}

For control subjects, isoeccentricity retinotopic maps were obtained using binocular, full-field stimuli (Figure 4A), which showed normal retinotopic organization in V1 (Figure 4B). For subjects with albinism, retinotopic maps (Figure 4C) were acquired using monocular hemifield stimuli (Figure 4A) as described previously (Hoffmann et al., 2003). In all subjects, the most complete and contiguous retinotopic activation was produced in the hemisphere contralateral to the visual stimulus, as would normally be expected (Figure 4C, columns 1 and 4; see also data for the left eye in Supplemental Figure S1). However, each hemisphere could also be activated by the ipsilateral visual field, which resulted in overlaid representations of both hemifields within the same hemisphere, a highly aberrant result (Figure 4C, columns 1 and 3 for the left hemisphere, columns 2 and 4 for right hemisphere). These aberrant hemifield representations were asymmetric, with the most extensive activation in the hemisphere contralateral to the stimulated eye. Thus, when the right eye was stimulated (as in Figure $\mathbf{4 C}$ ) the aberrant 
407 activation of the left hemisphere was more prominent than that in the right hemisphere

408 (Figure 4C, column 3 greater than column 2), and when the left eye was stimulated the

409 aberrant activation of the right hemisphere was more prominent than that in the left

410 hemisphere (Supplemental Figure S1, column 2 greater than column 3). This asymmetry

411 is also evident when comparing the normal activation to the aberrant activation in the

412 same hemisphere: for example, in subject JC_0492, when the right eye was stimulated

413 the normal and aberrant representations in the left hemisphere (i.e., contralateral

414 hemisphere; Figure 4C, columns 1 and 3) appeared more similar than the two

415 representations in the right hemisphere (i.e., ipsilateral hemisphere; Figure 4C, columns

4162 and 4). This was a general trend across subjects with albinism. There was a similar

417 trend for the left eye stimulus, with the greatest similarity between normal and aberrant

418 representations in the right hemisphere (see Supplemental Figure S1, where columns 2

419 and 4 were more similar to each other than columns 1 and 3 ).

In addition to these trends, however, there was also significant variation across our

421 albinism cohort (compare down each column of Figure 4C), which was particularly

422 evident with respect to the overall continuity of the isoeccentricity maps. This can be seen

423 clearly in subjects JC_0492 and JC_10227, who showed some of the most and least

424 contiguous maps: JC_0492 had a nearly complete retinotopic representation with few

425 holes (especially in the left hemisphere), while JC_10227 showed only sparse activation

426 in either hemisphere when the right visual field was stimulated. Additionally, JC_10227

427 showed notable activation by the peripheral visual field in the ipsilateral hemisphere

428 regardless of which hemifield was stimulated, which was not as prominent in other

429 subjects (Figure 4C, second column). 
Subjects in our albinism cohort also varied considerably in the predominance of central versus peripheral field representations (note dominant colors in each subject in Figure 4C). This is illustrated by directly comparing subjects JC_0493 and JC_10093: JC_0493 had much more central (red/orange) activation than JC_10093, but JC_10093 had greater peripheral (blue) activation than JC_0493. Additionally, subjects varied in the spatial localization of the isoeccentricity bands: subjects who had weak foveal activation also had peripheral activation that was apparent nearer to the occipital pole, where the

437 foveal confluence is typically found (compare the location of the yellow band across 438 subjects in Figure 4 C). In general, the extent of activation by the central visual field appeared to correlate with subjects' peak cone densities (shown below each row of 440 images in Figure 4C), but JC_10230 was a notable exception. This subject had the lowest

441 peak cone density but showed much clearer activation by central visual field than 442 JC_10093, who had the next-lowest peak cone density. mapping functions for representative controls and subjects with albinism are shown in

447 Figure 5A-B (control) and Figure 5E-F (albinism). The empirical data are shown by the 448 red and blue points for the right and left hemispheres, respectively (and for the 3 ROls 449 within each hemisphere). Fitted curves (equation 3, Methods) are shown as the smooth 450 colored lines (See Supplemental Figure S2 for data/curves for all subjects). The fitted 451 curves for all subjects are shown in Figure 5C and 5G. When the map scaling factor, $c$, 452 from the fitted cortical mapping functions was compared across hemispheres, there was 
453 no significant difference between the right and left hemispheres (Wilcoxson matched454 pairs test, $p>0.99)$.

In subjects with albinism, each visual hemifield in each eye was stimulated 456 separately, resulting in eight possible cortical mapping functions for each subject (2 457 hemispheres $\times 2$ visual hemifields $\times 2$ eyes). However, due to the limited extent of 458 activation by the ipsilateral hemifield in either hemisphere, Figure 5E-H shows only the 459 curves representing activation by the contralateral visual hemifield. Since each eye was 460 stimulated separately, the cortical mapping functions from each eye were compared 461 within each hemisphere by comparing the map scaling factors ( $c$ from equation (3)). The 462 functions from the right and left eyes were not significantly different (paired $t$-test: $t=0.24$, $463 \mathrm{df}=9, p=0.82)$, and the functions from the same eye were not significantly different 464 between hemispheres (paired $t$-test: $t=0.42$, df $=9, p=0.68$ ). Mapping functions were 465 also compared between controls and subjects with albinism (Figure 5C versus 5G). 466 When the map scaling factors for all control subject hemispheres (mean \pm SD: $0.0587 \pm$ 467 0.0086) were compared to those for subjects with albinism (mean \pm SD: $0.0566 \pm 0.0188$ ), 468 there was no significant difference (Mann-Whitney $U$-test: $U=82, p=0.44$ ). The size of $\mathrm{V} 1$ is known to directly impact the magnitude of estimated CM, though 470 the shape of the function does not change significantly (Sereno et al., 1995). In order to 471 verify that the trend toward smaller V1 surface area in subjects with albinism did not also 472 affect the above comparison between control and albinism groups, the map scaling factor 473 in each task was compared to the V1 surface area of the corresponding hemisphere. The 474 map scaling factors were not significantly correlated with V1 surface area in either control 
$475\left(r^{2}=0.22, p=0.17\right)$ or albinism groups (right eye: $r^{2}=0.27, p=0.19 ;$ left eye: $r^{2}=0.28$, $476 p=0.17)$.

The fitted cortical mapping functions were then used to derive the cortical 478 magnification function (CMF) for each task in each subject (see Equation (4) in Methods).

479 The resulting functions for control and albinism subjects are shown in Figure 5D and 5H 480 respectively. CMFs for control subjects were modeled separately for each hemisphere.

481 For subjects with albinism, CMFs were modeled separately for each eye and each 482 hemisphere (stimulated by the contralateral visual field). When the CMF scaling factors 483 were compared between control subjects (mean \pm SD: $17.33 \pm 2.26$ ) and subjects with 484 albinism (mean \pm SD: $19.75 \pm 6.93$ ), there was no significant difference (Mann-Whitney $485 U$-test: $U=82, p=0.44)$. However, the CMF scaling factor was more variable in albinism 486 relative to controls ( $F$ test: $F=9.45, \mathrm{DFn}=19, \mathrm{Dfd}=9, p=0.002$ ). This is evident in 487 Figure 5D versus $5 \mathrm{H}$ (in $5 \mathrm{H}$, the dashed gray line is the average of the fitted curves from 488 control subjects, and the shaded gray area represents the values within 1 SD of the 489 average).

As mentioned above, all subjects with albinism had aberrant ipsilateral hemifield representations superimposed on the normal representation of the contralateral visual

492 field (Figure 4C). In most subjects this ipsilateral activation was highly truncated 493 compared to the contralateral activation. However, two subjects had at least one 494 hemisphere with sufficient ipsilateral activation to compare the aberrant activation to the 495 "normal" activation within the same hemisphere. Figure 6 shows the comparison between 496 the aberrant ipsilateral field mapping data (red) and the normal contralateral field mapping 497 data (gray) in these unique subjects. In JC_10093, the data from the two hemifields did 
498 not match, but rather the aberrant activation showed a unique pattern compared to the normal activation. When only the contralateral hemifield was stimulated, activation in the same hemisphere but from different eyes showed much better agreement (see Supplemental Figure S2 for comparison). In JC_10227, the aberrant activation appeared to agree with the normal activation overall. However, in the aberrant activation there was notable variability between ROls, particularly in the peripheral visual field, which was not as pronounced in the normal activation.

Figure 7 shows empirically-derived CMFs (red) for each subject with albinism

508 along with a predicted CMF (green) based on cone density alone. Control results are shown in Figure 8. As outlined in Methods, the cone density predictions have been 510 intentionally aligned to the empirically-based curves at $8^{\circ}$ eccentricity, to facilitate 511 comparison of the shapes of the curves rather than their absolute differences (which are 512 sensitive to an arbitrary scaling factor). It is clear in all hemispheres for which the data 513 extend to within $2^{\circ}$ of the center of gaze (Figure 7, dark gray shaded region) that the 514 empirically measured CM increases far more rapidly than is predicted by cone density 515 alone, and this is also the case (albeit to a lesser extent) between $2-4^{\circ}$ eccentricity (Figure 5167 , light gray shaded region). This is true for both albinism (Figure 7) and control groups 517 (Figure 8).

518 In order to determine whether cortical organization was correlated with cone 519 density, the cortical mapping functions and CMFs were also compared to each subject's 520 peak cone density. Since cone density was measured in only one eye in each subject, 
521 only the cortical functions based on activation by the imaged eye (i.e., the right eye in all

522 subjects except JC_10230; see Table 1) was included in this analysis. The average map

523 scaling factor $(c)$ and the CMF scaling factor $(1 / c)$ from both hemispheres was plotted

524 against peak cone density for each subject. Neither the map scaling factor nor the CMF

525 scaling factor was significantly correlated with peak cone density in either control subjects

$526 \quad\left(c: r^{2}=0.053, p=0.71 ; 1 / c: r^{2}=0.067, p=0.67\right)$ or in subjects with albinism $\left(c: r^{2}=0.35\right.$,

$\left.527 p=0.29 ; 1 / c: r^{2}=0.17, p=0.49\right)$.

528

529

DISCUSSION

530

Aberrant Retinotopy in Albinism

The results of this study are largely consistent with previous reports of highly aberrant retinotopic organization in visual area V1 of subjects with albinism (Creel et al., 1974; Dorey et al., 2003; Hoffmann et al., 2003; Schmitz et al., 2004) but significantly extend those findings to include an analysis of individual variability and a quantitative account of CM compared with individual retinal cone density measurements. Aberrant retinotopic organization was apparent in all subjects with albinism and consisted of superimposed representations of opposite hemifield representations within the same

538 hemisphere. The aberrant (ipsilateral) hemifield activation was most prominent in the 539 hemisphere contralateral to the eye being stimulated. This aberrant activation typically 540 represented regions of the visual field within approximately $5-6^{\circ}$ of the center of gaze but 541 varied considerably across the albinism group. This agrees with previous studies that 542 found significant variation in the left-right margin of aberrant decussation among 543 individuals with albinism (Dorey et al., 2003; Hoffmann et al., 2005; von dem Hagen et 
544 al., 2007). Overall, these findings support previous descriptions of pathological optic 545 nerve decussation at the optic chiasm in albinism. Ganglion cell projections from the

546 temporal retina that ordinarily synapse in the ipsilateral hemisphere instead decussate to

547 the contralateral hemisphere (Creel et al., 1978; Hoffmann et al., 2003), giving rise to the 548 aberrant cortical retinotopy.

549 When the surface area of V1 was measured in this study, subjects with albinism 550 had modestly reduced surface area relative to control subjects, but this difference was 551 not statistically significant (Figure 3). This is not surprising, because changes in occipital 552 gray matter volume that were reported in previous studies of albinism were highly 553 localized to the occipital pole (von dem Hagen et al., 2005; Bridge et al., 2014); therefore, 554 measurements of V1 surface area as a whole are less likely to be dramatically affected. 555 However, subjects with albinism are also known to have reduced optic nerve, optic 556 chiasm, and optic tract size (von dem Hagen et al., 2005; Ather et al., 2019), which may 557 reflect a reduction in retinal afferents from the foveal region where cone density is reduced 558 (Wilk et al., 2014). In normal visual system development V1 surface area is thought to be 559 correlated with optic tract size (Andrews et al., 1997). Thus, the apparent lack of 560 correlation between previous reports of reduced optic tract size in albinism and our 561 measurements of nearly normal V1 surface area in albinism suggests that unique factors 562 involved in the development of retinocortical projections in albinism may preserve cortical 563 space despite a reduced number of retinal afferents. 
In the albinism group, we noted marked variations in features of the overall pattern of retinotopic organization that have not been appreciated previously. These features included both the continuity of the retinotopic maps and the relative surface area devoted to central versus peripheral visual field activation. Such variability within the albinism population is consistent with previous studies that have shown significant variability in

571 retinal phenotypes among individuals with albinism, particularly in the severity of foveal

572 hypoplasia (Thomas et al., 2011; Kruijt et al., 2018) and in peak cone packing density

573 (Wilk et al., 2014; Wilk et al., 2017). Indeed, our group previously performed a detailed

574 analysis of the retinal structure of the subjects presented in this study (Wilk et al., 2014;

575 Wilk et al., 2017). The results of that study were used to intentionally select subjects with

576 albinism for $\mathrm{fMRI}$ imaging who represented a broad spectrum of retinal structure (for cone

577 densities, see Figure 2).

Overall, the area of cortical activation corresponding to the central visual field in subjects with albinism appeared to be reduced compared to control subjects. This may be partly due to the visual task used for retinotopic mapping in subjects with albinism, because the smallest annulus in the expanding ring stimulus did not include the foveal center. Rather, the center of fixation was covered by the round fixation marker that had a 583 radius of $0.8^{\circ}$ and appeared randomly to control for attention. While the central activation

584 might have been improved by using a contracting ring or drifting bar stimulus (Dumoulin 585 and Wandell, 2008), the presence of robust foveal activation in some subjects with 586 albinism (e.g. JC_0492 and JC_0493) indicates that the stimulus itself was not sufficient 587 to prevent strong activation by central regions of the visual field. It is also possible that 
alertness during imaging, so we explicitly monitored alertness by self-report after every

fMRI scan. Only one subject (JC_0493) reported alertness levels below 3 (on a scale from

1 to 5 , where 5 is most alert), yet this subject had some of the most robust activation

patterns. Therefore, we do not believe the reduced foveal representation in albinism to be artifactual. Moreover, this observation is consistent with previous fMRI-based findings in albinism (Schmitz et al., 2004). As mentioned above, known structural changes in V1 in albinism are also preferentially found at the occipital pole (von dem Hagen et al., 2005; Bridge et al., 2014), which further corroborates an overall reduction in the functional activation of the foveal region.

We also found that the decreased foveal activation correlated with peak cone density in four of five subjects with albinism. However, the presence of one subject who did not fit this trend (JC_10230) indicates that factors other than cone density (for example, the divergence or convergence of retino-cortical projections) may also contribute to the relative sizes of the central and peripheral retinotopic representations.

The extent of central field activation across subjects with albinism tended to be

604 inversely correlated with the extent of peripheral field activation. That is, in subjects who 605 had reduced foveal activation the peripheral regions of the visual field tended to expand 606 toward the occipital pole (see Figures 4C and S1, columns 1 and 4). One possible 607 explanation for this phenomenon is that, when input from the central visual field is reduced 608 or absent during development, retino-cortical afferents representing more peripheral regions of the visual field may expand into cortical space normally allocated to the more central portions of the visual field. This would also explain our finding that $\mathrm{V} 1$ surface area

611 is relatively normal despite a known reduction in retinal afferents that is concentrated at 
612 the fovea (Wilk et al., 2014) and in optic tract size (von dem Hagen et al., 2005; Ather et

613 al., 2019). A similar cortical expansion of non-foveal afferents has been described

614 previously in achromatopsia. In this genetic disease there is a complete absence of

615 functional cone photoreceptors, yet stimulation of rod photoreceptors leads to aberrant

616 cortical activation near the occipital pole (Baseler et al., 2002). Thus, it is possible that

617 analogous cortical reorganization may occur in albinism, in which peripheral inputs

618 "spread out" into cortical regions that might otherwise be occupied by foveal afferents. A

619 second possible explanation is that a release of lateral inhibition by the visual cortical

620 neurons that are typically activated by the central visual field allows the neighboring

621 retinotopic areas to become more active, though it is unclear how such an effect might

622 propagate throughout the map to permit expansion of the most peripheral representation.

Cortical Magnification: Albinism vs. Controls subjects with albinism were not (on average) significantly different from those for control

627 subjects (Figure 5G; albinism curves shown in orange, average control function shown 628 in gray). Our control data also match those of Sereno et al. and Engel et al. (Sereno et 629 al., 1995; Engel et al., 1997). This similarity between control subjects and subjects with 630 albinism was also evident when the fitted cortical mapping functions were used to model 631 the CMF in each hemisphere (Figure $5 \mathbf{H}$ ). This allows us to reject our initial hypothesis 632 that reduced cone density in albinism would necessarily lead to reduced CM. Instead, 633 some of the hemispheres of subjects with albinism tended to have greater $\mathrm{CM}$ in 634 peripheral regions of the visual field compared to the control group (Figure $\mathbf{5 H}$, orange 
635 curves outside the gray underlay representing controls). This trend in CM would not be explained by overall surface area of $\mathrm{V} 1$, because (as discussed above) the surface area

637 of V1 was not significantly different in albinism than in controls, and in fact trended toward 638 decreased $\mathrm{V} 1$ surface area. This suggests that, in albinism, retinal cone density does not 639 determine the size of the retinotopic representation in $\mathrm{V} 1$ as has previously been 640 suggested (Dougherty et al., 2003), but rather that the cones that are present (along with 641 their downstream synaptic partners) capitalize on the entirety of the cortical space 642 available to them. In this scenario, it is possible that the amount of cortical space devoted 643 to V1 (and other visual areas) is primarily determined by non-retinal factors, which may 644 be unique to albinism (or similar genetic conditions).

645 It is also notable that, for one of the subjects with albinism (e.g. JC_10093), the 646 cortical eccentricity mapping functions for the normal and aberrant fields had local zones

647 in which the eccentricities differed markedly despite being represented by exactly the 648 same cortical voxels (Figure 6). This indicates that the two hemifield representations are 649 not always precisely in mirror-image register, and that more subtle wiring anomalies can 650 occur in addition to those related to a shift in the line of left-right decussation at the optic 651 chiasm. Though one might suppose that these more subtle errors simply represent 652 random variations, it is important to appreciate the precision of same hemifield overlap in 653 normal controls that gives rise to the systematic and precise computations of retinal 654 disparity responsible for stereopsis. Whether the lack of precise registration between 655 opposing hemifield representations in albinism is functionally important remains unclear. 
Cortical Magnification: Empirical vs. Cone-Based Predictions alone, assuming that each cone was allocated an equal amount of cortical space in V1. All subjects—both controls and albinism—showed a greater increase in CM near the center of the visual field than would be predicted based on cone density alone (Figures

7, 8). When the empirical and cone-predicted CMFs are compared in the central-most

664 regions (see shaded areas in Figure 7), it is evident that both the magnitude and slope of the empirical functions (red) are greater than the cone-based predictions (green). Note, however, that the empirical data within the central 2 degrees (dark gray zone) are marginal due to limitations of the stimulus (see Methods), and this was particularly true for two of our subjects with albinism (JC_10093, JC_10227). This is important because

669 one might expect that the extreme loss in cone density observed in albinism relative to control subjects would lead to markedly reduced CMFs. However, the biggest differences

671 in cone density are limited to the fovea and don't extend beyond $2^{\circ}$ where our more 672 reliable $\mathrm{CM}$ data begin. This might also account for the failure to observe major 673 differences in CMF between subjects with albinism and controls.

$674 \quad$ That empirical CM values exceed the cone-based predictions might be explained 675 by a number of factors. First, it is known that there is greater convergence of cones onto 676 peripheral retinal ganglion cells (RGCs) compared to central RGCs (Curcio and Allen, 677 1990; Dacey and Petersen, 1992; Dacey, 1993), which would exacerbate cone-based 678 differences in central versus peripheral magnification. Moreover, RGCs with receptive 679 fields near the center of the visual field are thought to project to more cortical space than 
680 peripheral RGCs (Azzopardi and Cowey, 1996; Popovic and Sjöstrand, 2001), though 681 this is debated (Wässle et al., 1990). In order to examine the representation of RGCs in $682 \mathrm{~V} 1$, one must consider both the convergence/divergence of GC projections onto LGN 683 neurons and divergence of LGN projections on to layer 4 of V1. Indeed, Azzopardi and 684 Cowey (Azzopardi and Cowey, 1996) argue that the final V1 cortical magnification 685 function is the result of successive expansion of the foveal representation both in the LGN and in the cortex. The stage between the LGN and cortex was described in detail by 687 Connolly and Van Essen (Connolly and Van Essen, 1984) for the macaque monkey. They 688 found that "The total number of cortical neurons per LGN neuron is about 130 on average, but it extends over approximately a tenfold range, from less than 100 in the far periphery 690 to nearly 1,000 in the fovea." Whether this tenfold difference in divergence is also true 691 for humans with or without albinism is unknown, but is likely to be a major factor in 692 determining the resulting cortical magnification. Clearly, a comprehensive, quantitative 693 account of the neural basis of CMF must await more extensive estimates of 694 convergence/divergence versus eccentricity at each stage of the retinostriate hierarchy.

\section{Behavioral Predictions}

Given previous observations that human visual acuity is normally correlated with CM (Duncan and Boynton, 2003), the similarities in CMFs between controls and subjects 699 with albinism in this study might predict that parafoveal and peripheral visual acuity would 700 be similar in albinism to that in normal controls. A previous study of acuity in albinism 701 found that central visual acuity in albinism was reduced but peripheral visual acuity was 702 similar to normal controls, which supports this prediction (Wilson et al., 1988). However, 
703 that study only measured acuity at the center of gaze and at $10^{\circ}$ inferior, so it is currently

704 unknown at what point between the fovea and $10^{\circ}$ the acuity thresholds in albinism

705 approach normal levels. While our measurements of CM were limited to $2-16^{\circ}$ (i.e., they

706 did not extend to the fovea), the empirically-derived CMFs in subjects who had the most

707 extensive central representations appear to remain within normal limits (see Figure $\mathbf{5 H}$ ).

708 If this trend continues all the way to the fovea, it would indicate that the CMFs may not

709 necessarily account for the reduced central visual acuity typically observed in albinism.

710 However, the variability in cortical organization shown in this study indicates a role for

711 plasticity in modifying the functional relationship between retinal and cortical structures.

712 More detailed studies of visual acuity are needed in this population along with targeted

713 investigations of the cortical foveal confluence in order to further explore the etiology of

714 the visual acuity deficits in albinism (Azzopardi and Cowey, 1996).

715

$716 \quad$ Limitations

717 One of the primary limitations of this study is the small sample size. Both the rarity

718 of the disease and the prevalence of moderate to severe nystagmus within this population

719 serve as barriers to recruitment of large numbers of subjects with albinism who are good

720 candidates for fMRI. However, the finding that there is significant variability in retinotopic

721 organization between individuals with albinism only increases the importance of

722 measuring cortical phenotypes in more individuals to determine the full extent of

723 phenotypic variability. While we aim to increase our sample size in the future, the current

724 small cohort precludes observation of strong correlations between genetic subtypes of

725 albinism and distinct cortical phenotypes. Learning more about such genotype/phenotype 
726 correlations in albinism will be essential for guiding clinical diagnosis and for developing

727 interventional therapies.

728 An important methodological limitation of the current study is the use of expanding

729 checkered annuli to assess the eccentricity dimension of cortical retinotopy. The presence

730 of a relatively large fixation marker and the limited resolution of our visual stimulus display

731 within the MRI scanner compromised our ability to obtain reliable mapping data with

732 approximately 1.5 degrees of fixation. Future use of drifting bar stimuli (Dumoulin and

733 Wandell, 2008) in conjunction with an improved video display and fixation marker should

734 ameliorate this problem.

735 Another potential concern is that nystagmus and eccentric fixation are common in

736 albinism and might adversely affect the retinotopic maps. While nystagmus can introduce

737 noise into the fMRI signal, it is less likely to systematically alter the spatial properties of a

738 retinotopic map (Baseler et al., 2002). Moreover, BCEA values comparable to those

739 reported here for all but one subject with albinism do not appear to be correlated with

740 population receptive field $(\mathrm{pRF})$ sizes in other populations with unsteady fixation

741 (Clavagnier et al., 2015). Eccentric fixation, on the other hand, can affect the shape and

742 symmetry of cortical mapping functions (Baseler et al., 2002). However, the cortical

743 mapping functions that we observed tended to be notably symmetric between

744 hemispheres suggesting that eccentric fixation is unlikely to have had a significant effect

745 on the data presented here. 
This study both confirms previous findings of abnormal retinotopic organization in

749 albinism and expands upon these findings by showing that there is greater diversity in

750 retinotopy between individuals with albinism than has previously been appreciated. While

751 these changes often correlate with retinal cone density, this is not always the case.

752 Cortical magnification outside the fovea is not significantly different in albinism than in

753 normal controls and is greater in both groups than is predicted by cone density alone.

754 This indicates that post-receptoral mechanisms responsible for $\mathrm{CM}$ in albinism are at least

755 qualitatively, if not quantitatively, similar to those in normal controls.

756 Overall, our results show that the pattern of retinocortical miswiring that has

757 previously been ascribed to aberrant left-right decussation at the optic chiasm is

758 significantly more complex and varied than previously thought. Whether this additional

759 complexity occurs at the optic chiasm or represents additional connectivity changes

760 downstream is unclear. Albinism provides an excellent model in which both peripheral

761 and central effects of genetic mutation can be explored quantitatively. 

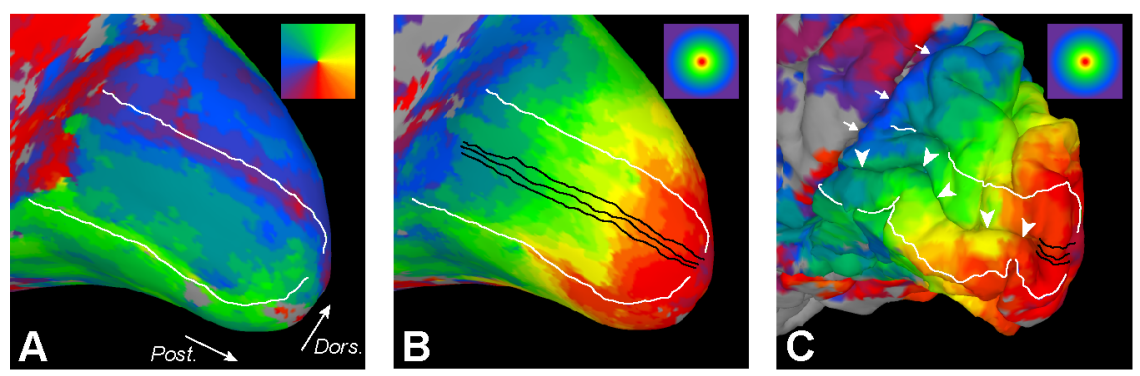

764 Figure 1: Medial occipital lobe retinotopic maps and sampling ROls displayed on cortical surface model for a representative control subject. Color coding for polar angle and eccentricity are shown in the upper-right corner of panels $A$ and $B$ respectively. (A) V1/V2 boundaries (white) associated with representations of the superior and inferior vertical

768 meridia (green and purple-red, respectively). White arrows indicate posterior (Post.) and

769 dorsal (Dors.) orientation and apply to all panels. (B) ROls (black) used to compute

770 cortical mapping functions oriented parallel to the representation of the horizontal

771 meridian within the calcarine sulcus (not shown). Phase-encoded eccentricity values

772 (surface coloring) were assigned to each node along the linear ROIs. (C) Linear distances

773 along the ROIs (buried within the calcarine sulcus) were calculated from the pial surface

774 of the original folded 3D surface model (not inflated). White arrowheads indicate the

775 calcarine sulcus. White arrows indicate parieto-occipital sulcus. 


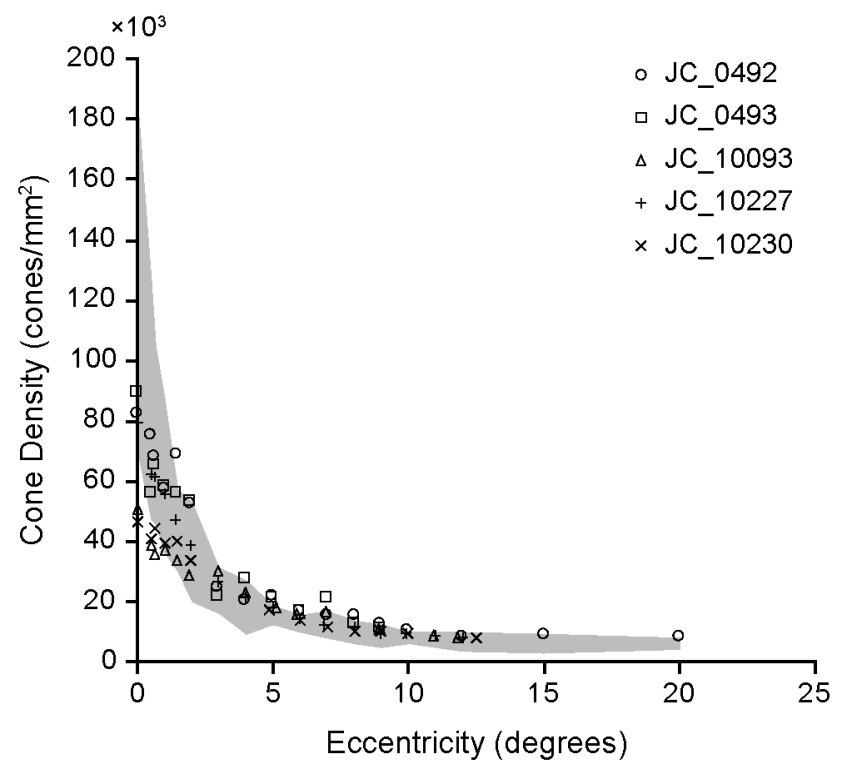

778 Figure 2: Retinal cone density as a function of eccentricity. Each subject with albinism is 779 represented by a different data point symbol. Gray shaded area represents the average 780 for all control subjects \pm 2 SD. 
A

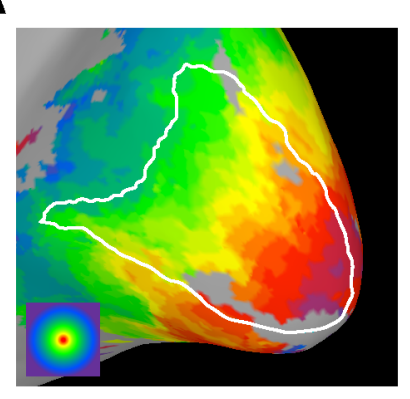

B

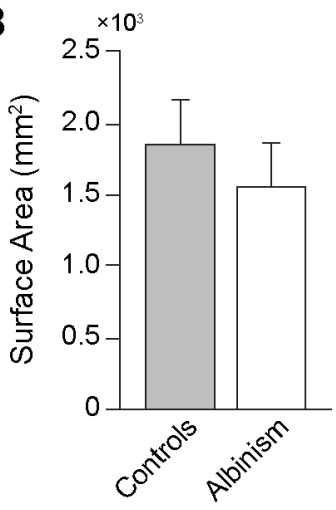

783 Figure 3: Mean surface area and gray matter volume of $\mathrm{V} 1$ is not significantly different

784 for albinism and control subjects (two-tailed $t$ test: $t=1.98, d f=16, p=0.065$ ). Boundaries used to compute V1 shown in Figure 1. Error bars represent one standard deviation.

786 Values for subjects with albinism are from only four subjects due to incompleteness of

787 cortical maps in one subject (JC_10230). 
A

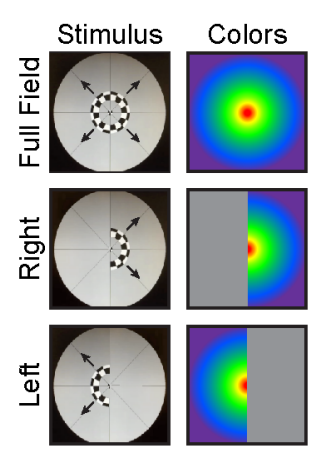

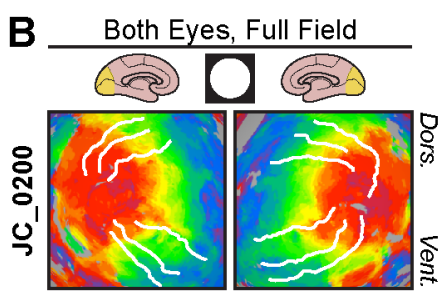

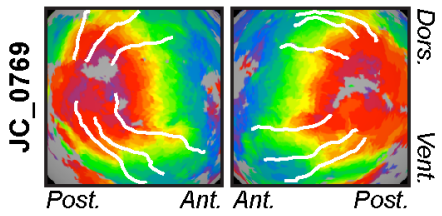

C Right Eye, Right Hemifield

Right Eye, Left Hemifield
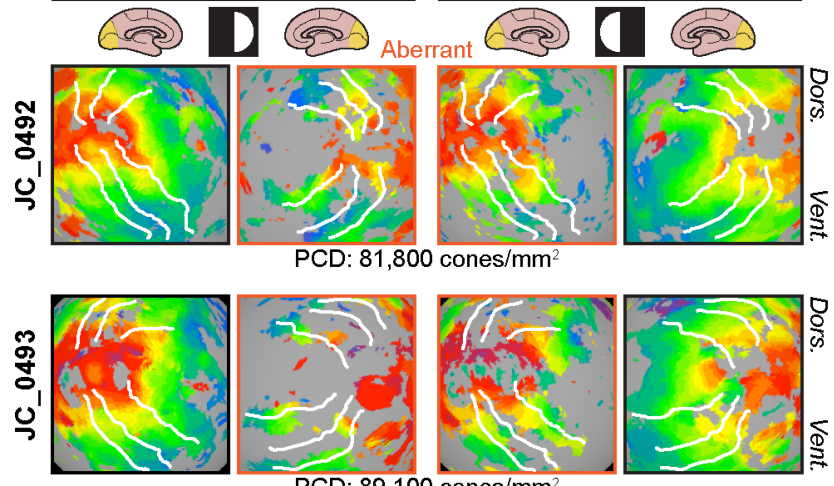

PCD: 89,100 cones $/ \mathrm{mm}^{2}$
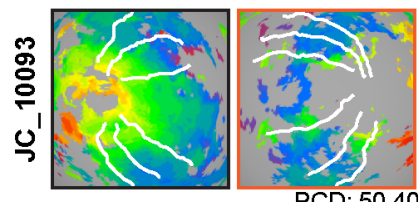

PCD: 50,400 cones $/ \mathrm{mm}^{2}$
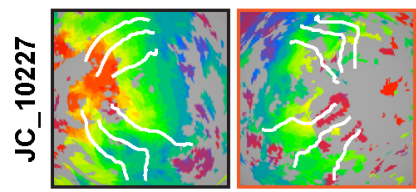

PCD: 78,900 cones $/ \mathrm{mm}^{2}$

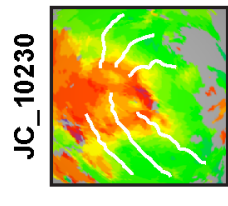

Post.

Ant. Ant.
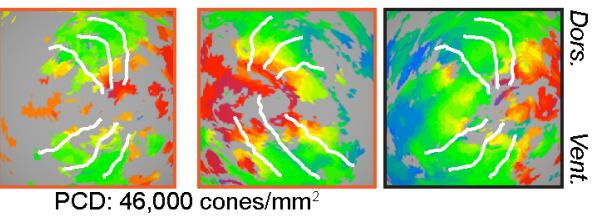

Ant. Ant.

790 Figure 4: Retinotopic maps of visual field eccentricity. (A) Visual stimuli and color coding

791 used for eccentricity mapping in controls (full field) and subjects with albinism (hemifield stimuli). (B) Eccentricity maps for two representative control subjects. All control subjects 
794 for subjects with albinism, obtained using hemifield ring stimuli viewed monocularly. Data 795 for right eye viewing condition are shown here (see Supplemental Figure S1 for left eye

796 data). Retinotopy patterns outlined in orange (middle columns) are aberrant ipsilateral

797 hemifield representations. Peak cone densities for each subject with albinism are 798 indicated below each row. All retinotopic maps are displayed on spherically-inflated 799 cortical surface models. Visual stimuli are indicated by white circle/semicircle symbols at 800 head of respective columns. White lines in B, C mark dorsal and ventral boundaries of

$801 \mathrm{~V} 1 / 2 / 3$ based on polar angle data (cf. Figure 1). PCD = peak cone density; Dors. = dorsal;

802 Vent. $=$ ventral; Ant. $=$ anterior; Post. $=$ posterior .

803 
804
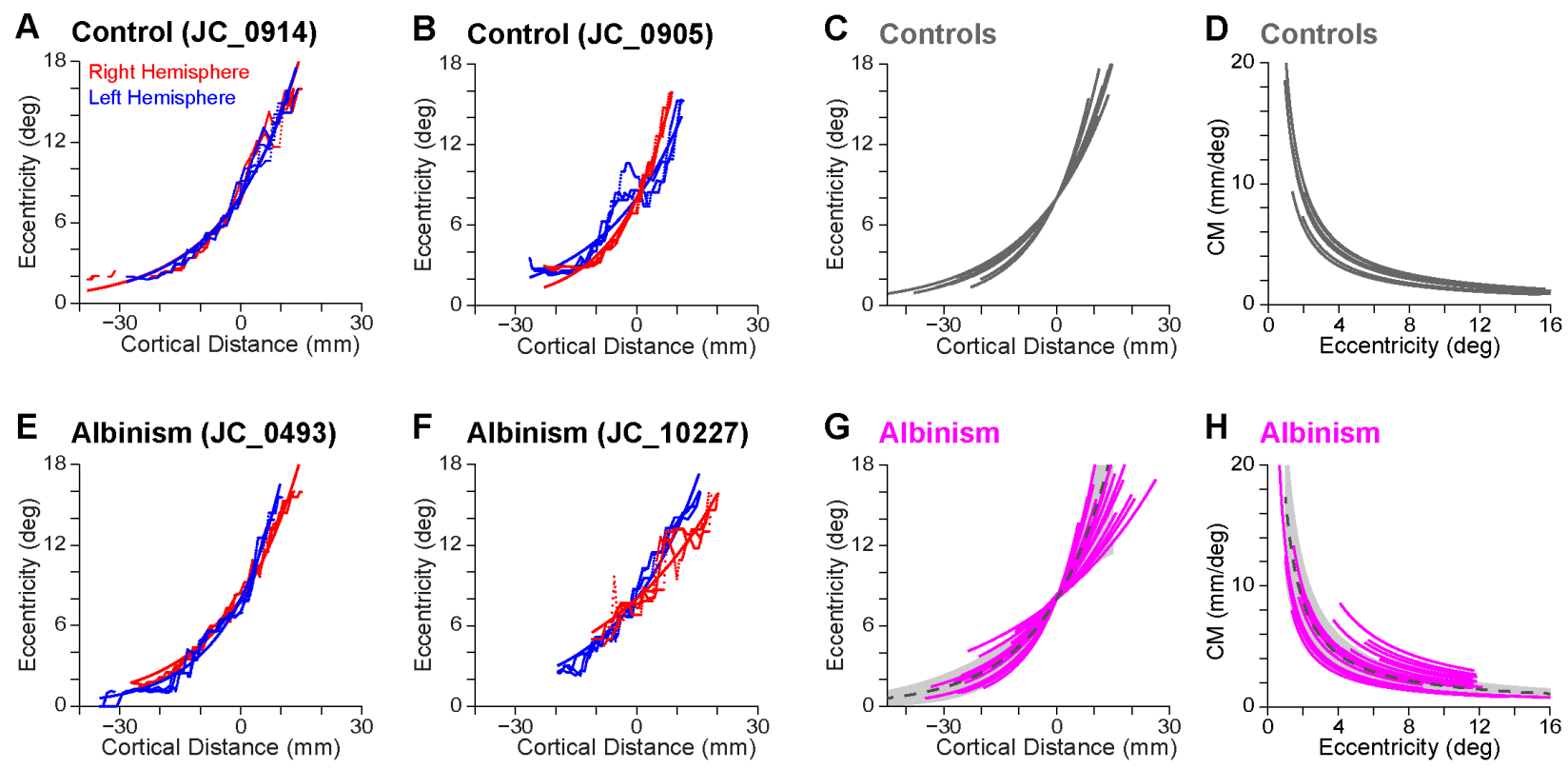

806 Figure 5: Cortical mapping and magnification functions in controls and subjects with

807 albinism. (A-B) Cortical mapping data and fitted functions for two representative control

808 subjects, including data from the left (blue) and right (red) hemispheres. (E-F) Cortical

809 mapping data and fitted functions for two representative subjects with albinism. Data for

810 left hemisphere (blue) obtained with right eye, right hemifield stimulation. Data for right

811 hemisphere (red) obtained with left eye, left hemifield stimulation. (C) Fitted cortical

812 mapping functions and (D) corresponding CMFs (see Methods) for both hemispheres of

813 all control subjects (gray curves). (G) Fitted cortical mapping functions and $(\mathrm{H})$

814 corresponding CMFs for subjects with albinism (magenta curves). Dashed dark gray lines

815 and gray shaded regions show mean \pm 1 SD for controls. In G and H data are combined

816 for left hemisphere, right hemifield stimulus and right hemisphere, left hemifield stimulus

817 and for both right and left eye viewing conditions. 
A JC_10093

Left Hemisphere, Right Eye

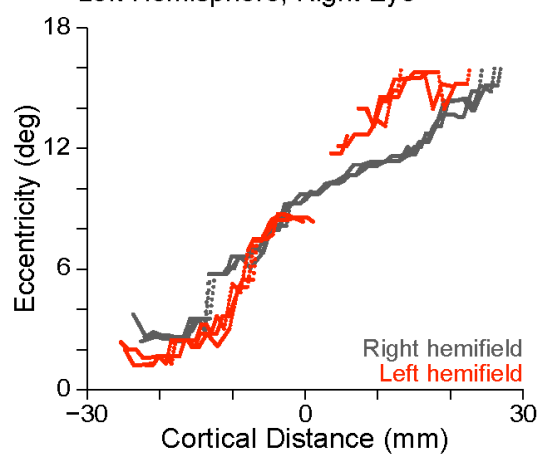

B JC_10093

Right Hemisphere, Left Eye

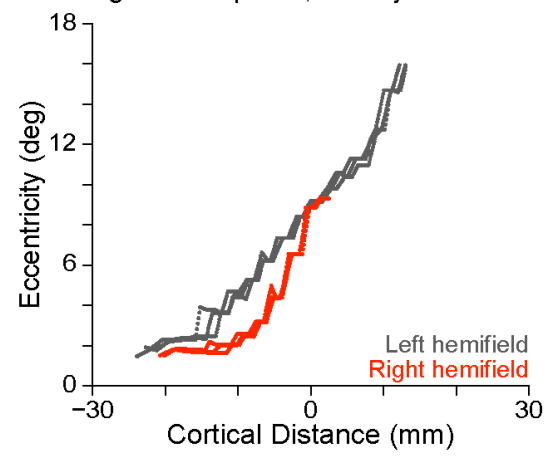

820 Figure 6: Comparison of empirical cortical mapping functions for normal contralateral

821 (gray) versus aberrant ipsilateral (red) hemifield stimulation in left and right hemispheres

822 of subject JC_10093. The red and gray data are from the same physical ROIs yet are

823 distinctly different. $\mathrm{LH}=$ left hemisphere; $\mathrm{RH}=$ right hemisphere. 


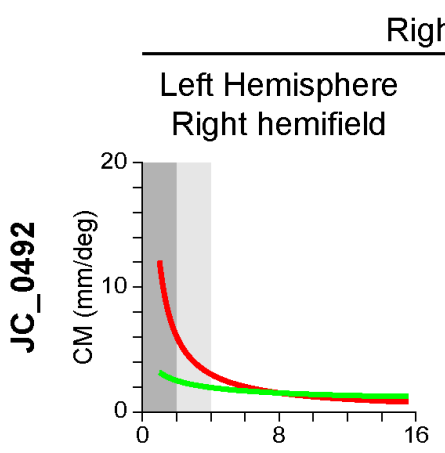

Right Eye
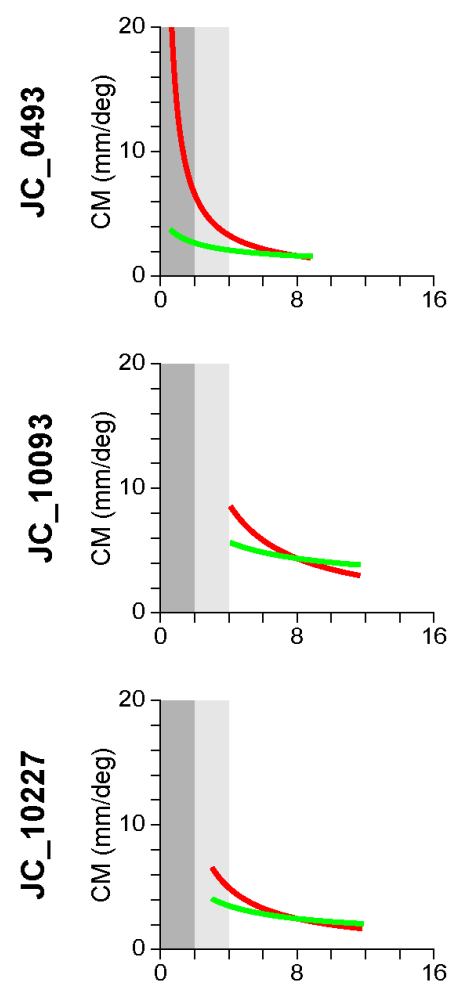

826

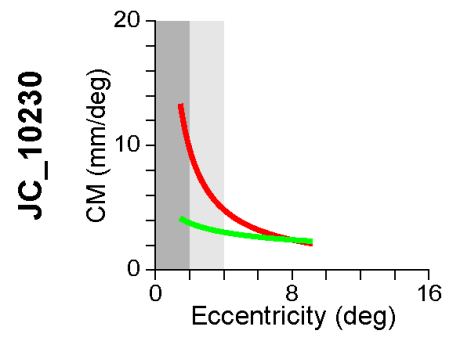

Right Hemisphere Left hemifield
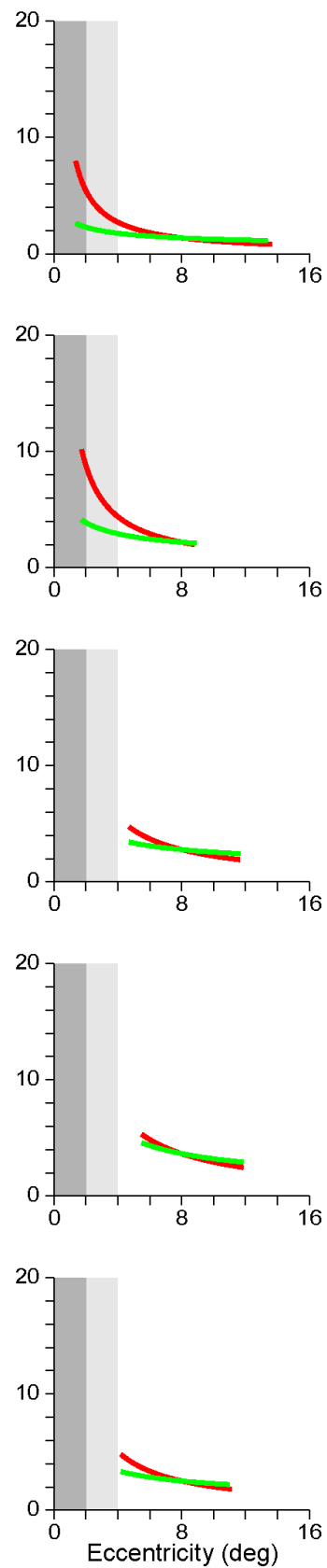
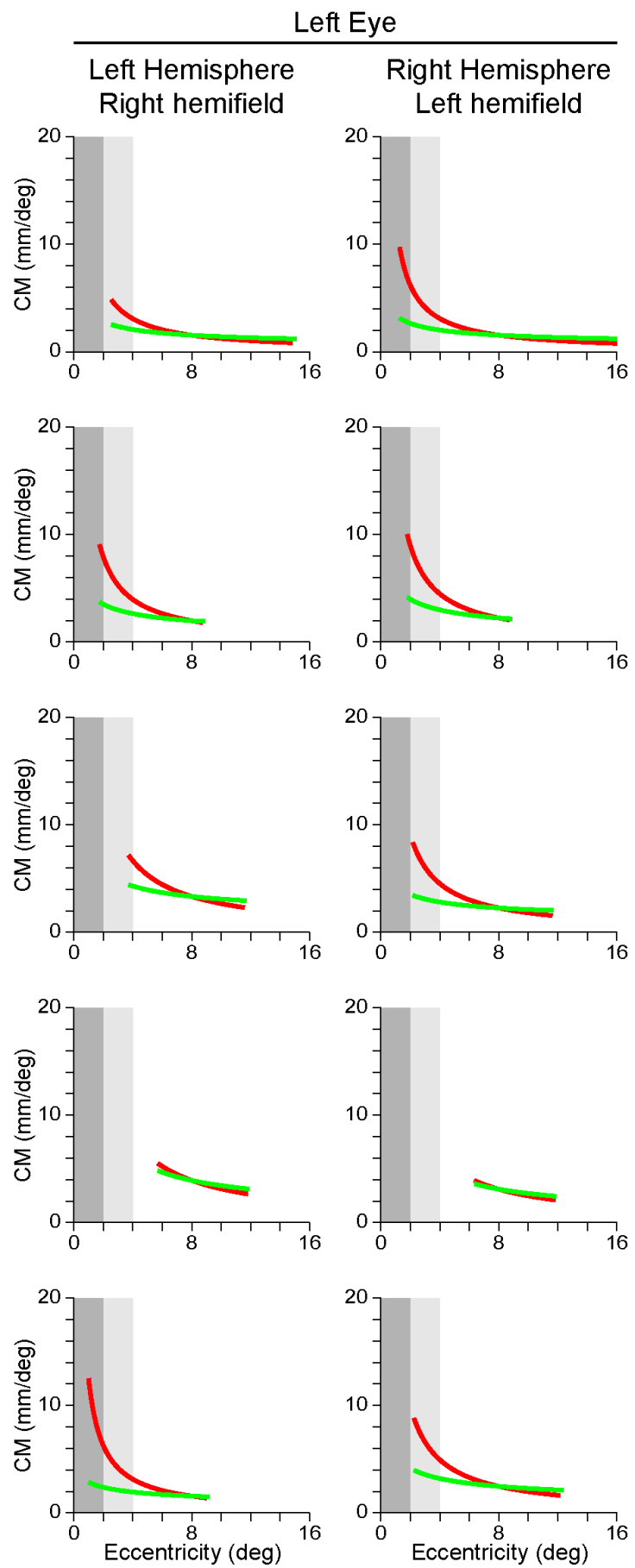

827 Figure 7: Cortical magnification functions based on empirical data (red) and cone density 

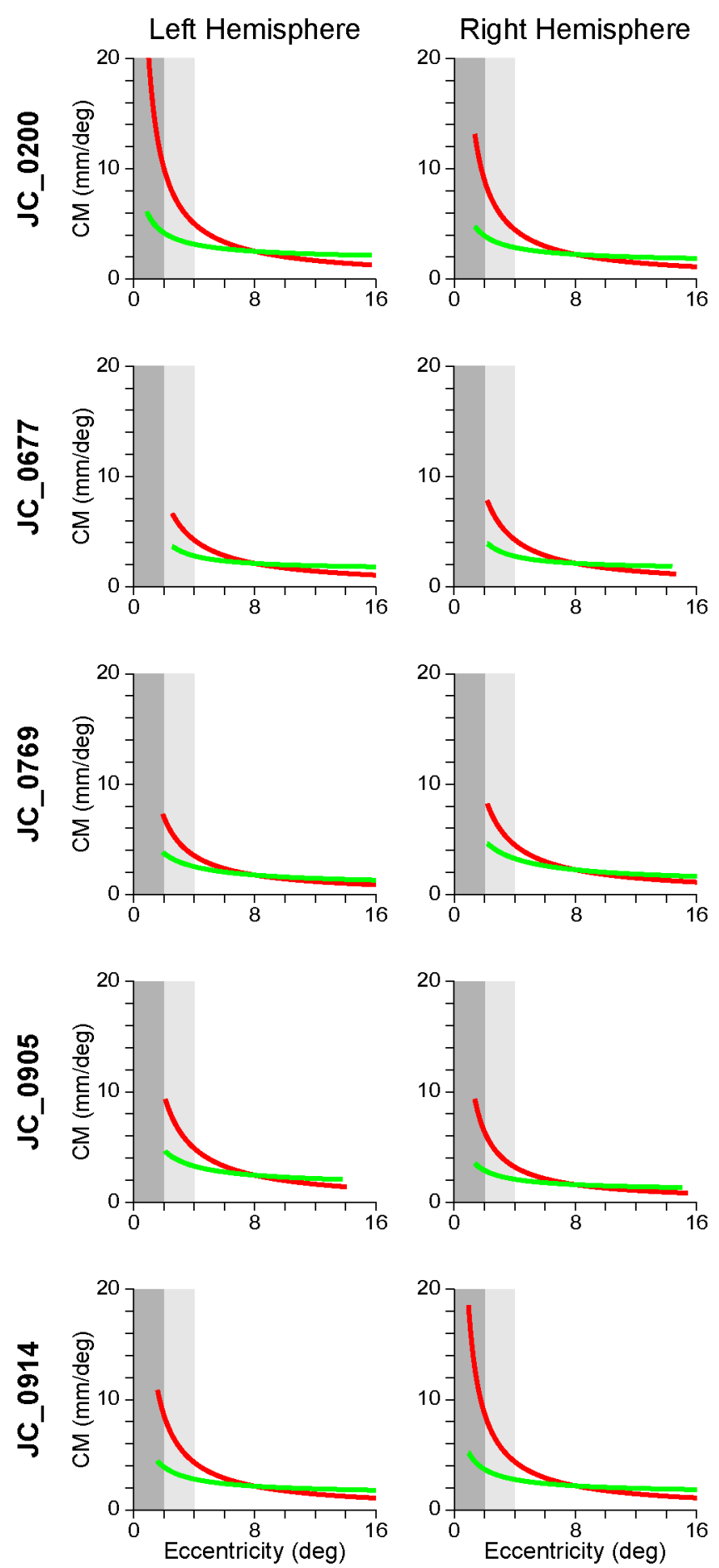

831 Figure 8: Cortical magnification functions based on empirical data (red) and cone density

832 predictions (green) for control subjects. To aid comparison, eccentricity ranges from $0-2^{\circ}$

833 and $2-4^{\circ}$ are shaded in dark and light gray respectively. 

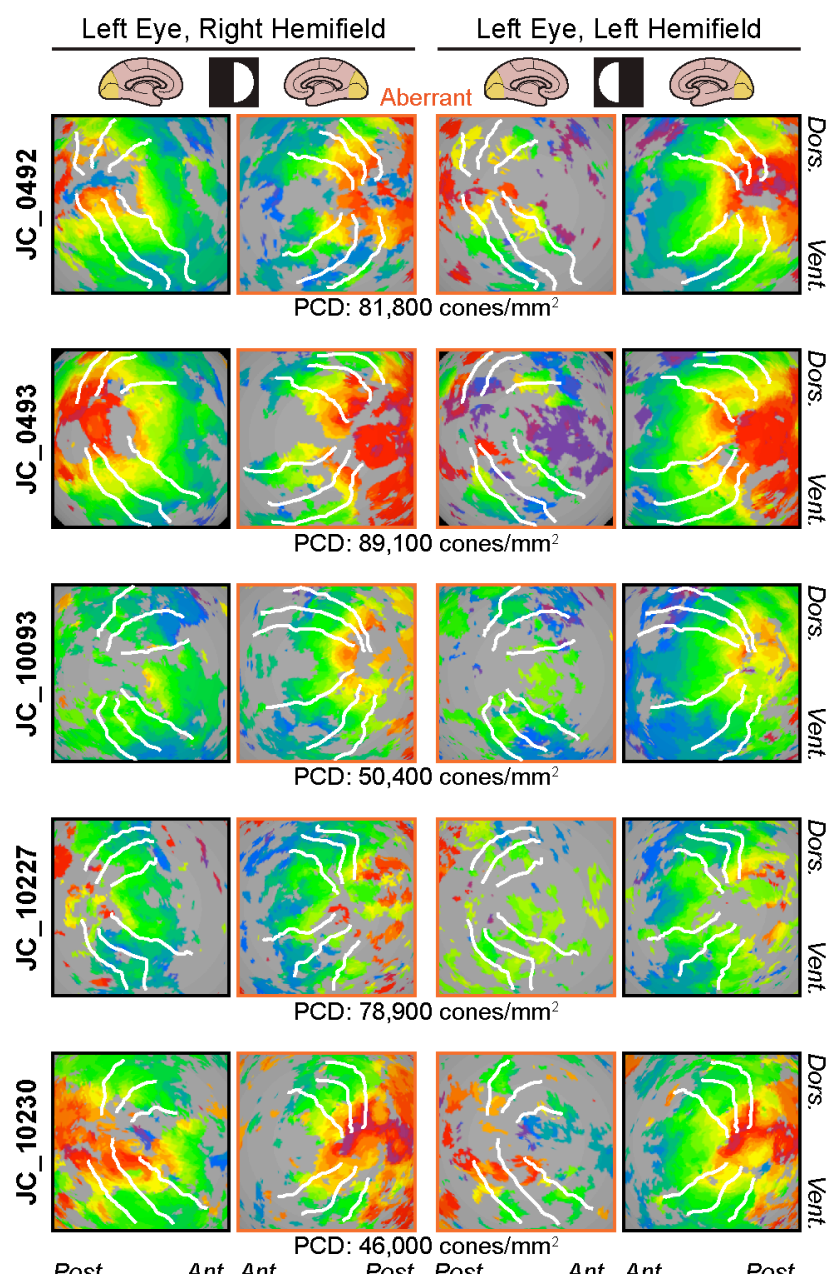

835 Supplemental Figure S1: Retinotopic maps of visual field eccentricity for left eye viewing condition in subjects with albinism. Visual stimulus and color coding as shown in Figure 4A. Retinotopy patterns outlined in orange are aberrant ipsilateral hemifield representations. Peak cone densities for each subject with albinism indicated below each row. Maps are displayed on spherically-inflated cortical surface models. Visual field

840 stimuli are indicated by white circle or semicircle symbols at head of respective columns.

841 White lines mark dorsal and ventral boundaries of $\mathrm{V} 1 / 2 / 3$ based on polar angle data (cf.

842 Figure 1). PCD = peak cone density; Dors. = dorsal; Vent. = ventral; Ant. = anterior; Post. $843=$ posterior . 

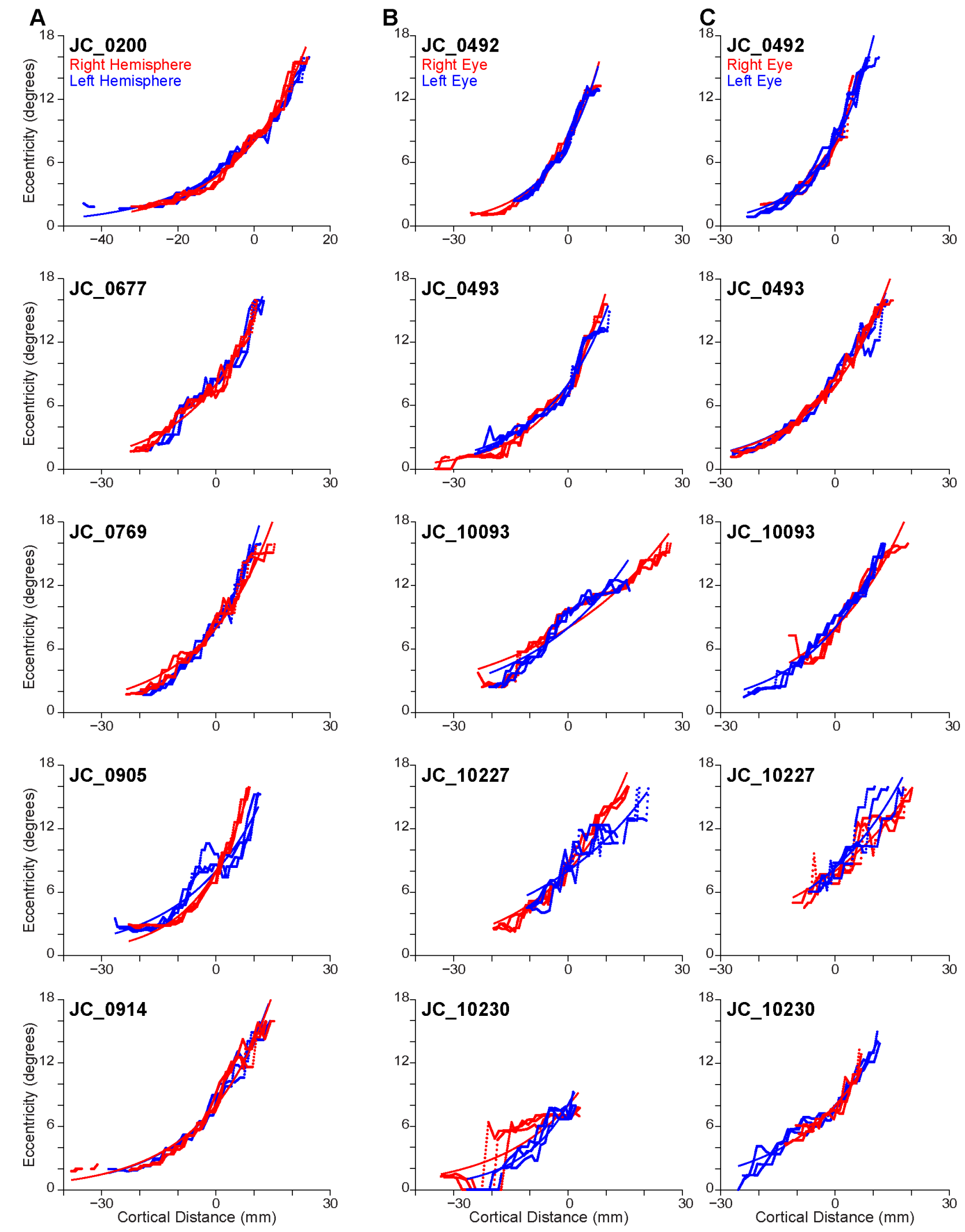
846 Supplemental Figure S2: Empirical cortical mapping data and fitted functions for all

847 subjects. (A) Controls: right hemisphere (red) and left hemisphere (blue). (B) Albinism:

848 left hemisphere, right hemifield stimulus. (C) Albinism: right hemisphere, left hemifield

849 stimulus. Viewing conditions in B, C: right eye (red), left eye (blue).

850 


\section{REFERENCES}

852 Andrews TJ, Halpern SD, Purves D (1997) Correlated size variations in human visual cortex, lateral geniculate nucleus, and optic tract. Journal of Neuroscience 17:2859-2868.

Ather S, Proudlock FA, Welton T, Morgan PS, Sheth V, Gottlob I, Dineen RA (2019) Aberrant visual pathway development in albinism: From retina to cortex. Human Brain Mapping 40:777-778.

Azzopardi P, Cowey A (1996) The overrepresentation of the fovea and adjacent retina in the striate cortex and dorsal lateral geniculate nucleus of the macaque monkey. Neuroscience 72:627-639.

Bandettini PA, Jesmanowicz A, Wong EC, Hyde JS (1993) Processing strategies for timecourse data sets in functional MRI of the human brain. Magnetic Resonance in Medicine 30:161-173.

Baseler HA, Brewer AA, Sharpe LT, Morland AB, Jägle H, Wandell BA (2002) Reorganization of

Bridge $H$, von dem Hagen EA, Davies G, Chambers C, Gouws A, Hoffmann MB, Morland AB (2014) Changes in brain morphology in albinism reflect reduced visual acuity. Cortex 56:64-72. human cortical maps caused by inherited photoreceptor anomalies. Nature

Connolly M, Van Essen D (1984) The representation of the visual field in parvicellular and

Clavagnier S, Dumoulin SO, Hess RF (2015) Is the cortical deficit in amblyopia due to reduced cortical magnification, loss of neural resolution, or neural disorganization? Journal of magnocellular laayers of the lateral geniculate nucleus in the macaque monkey. Journal of Comparative Neurology 226:544-564. 
883

Creel D, O'Donnell FE, Jr., Witkop CJ, Jr. (1978) Visual system anomalies in human ocular albinos. Science 201:931-933.

Crossland MD, Sims M, Galbraith RF, Rubin GS (2004) Evaluation of a new quantitative technique to assess the number and extent of preferred retinal loci in macular disease. Vision Research 44:1537-1546.

Curcio CA, Allen KA (1990) Topography of ganglion cells in human retina. Journal of Comparative Neurology 300:5-25.

Curcio CA, Sloan KR, Kalina RE, Hendrickson AE (1990) Human photoreceptor topography. Journal of Comparative Neurology 292:497-523.

Dacey DM (1993) The mosaic of midget ganglion cells in the human retina. Journal of Neuroscience 13:5334-5355.

Dacey DM, Petersen MR (1992) Dendritic field size and morphology of midget and parasol ganglion cells of the human retina. Proceedings of the National Academy of Sciences of the United States of America 89:9666-9670.

Daniel PM, Whitteridge D (1961) The representation of the visual field on the cerebral cortex in monkeys. Journal of Physiology 159:203-221.

Datta R, DeYoe EA (2009) I know where you are secretly attending! The topography of human visual attention revealed with fMRI. Vision Research 49:1037-1044.

DeYoe EA, Carman GJ, Bandettini P, Glickman S, Wieser J, Cox R, Miller D, Neitz J (1996) Mapping striate and extrastriate visual areas in human cerebral cortex. Proceedings of the National Academy of Sciences of the United States of America 93:2382-2386.

Dorey SE, Neveu MM, Burton LC (2003) The clinical features of albinism and their correlation with visual evoked potentials. British Journal of Ophthalmology 87:767-772.

Dougherty RF, Koch VM, Brewer AA, Fischer B, Modersitzki J, Wandell BA (2003) Visual field representations and locations of visual areas $\mathrm{V} 1 / 2 / 3$ in human visual cortex. Journal of Vision 3:586-598.

Dubra A, Harvey Z (2010) Registration of 2D images from fast scanning ophthalmic instruments. In: Biomedical Image Registration, 1st Edition (Fischer B, Dawant B, Lorenz C, eds), pp 60-71. Berlin: Springer-Verlag.

Dubra A, Sulai Y (2011) Reflective afocal broadband adaptive optics scanning ophthalmoscope. Biomedical Optics Express 2:1757-1768.

Dumoulin SO, Wandell BA (2008) Population receptive field estimates in human visual cortex. Neurolmage 39:647-660. 
Duncan RO, Boynton GM (2003) Cortical magnification within human primary visual cortex correlates with acuity thresholds. Neuron 38:659-671.

Engel SA, Glover GH, Wandell BA (1997) Retinotopic organization in human visual cortex and the spatial precision of functional MRI. Cerebral Cortex 7:181-192.

Fujii GY, De Juan E, Jr., Humayun MS, Sunness JS, Chang TS, Rossi JV (2003) Characteristics of visual loss by scanning laser ophthalmoscope microperimetry in eyes with subfoveal choroidal neovascularization secondary to age-related macular degeneration. American Journal of Ophthalmology 136:1067-1078.

Garrioch R, Langlo C, Dubis AM, Cooper RF, Dubra A, Carroll J (2012) Repeatability of in vivo parafoveal cone density and spacing measurements. Optometry and Vision Science 89:632-643.

Hendrickson A (2005) Organization of the adult primate fovea. In: Macular Degeneration (Penfold PL, Provis JM, eds), pp 1-20. Heidelberg: Springer-Verlag.

Hoffmann MB, Tolhurst DJ, Moore AT, Morland AB (2003) Organization of the visual cortex in human albinism. Journal of Neuroscience 23:8921-8930.

Hoffmann MB, Lorenz B, Morland AB, Schmidtborn LC (2005) Misrouting of the optic nerves in albinism: Estimation of the extent with visual evoked potentials. Investigative Ophthalmology \& Visual Science 46:3892-3898.

Kruijt CC, de Wit GC, Bergen AA, Florijn RJ, Schalij-Delfos NE, van Genderen MM (2018) The phenotypic spectrum of albinism. Ophthalmology 125:1953-1960.

Morland AB, Baseler HA, Hoffmann MB, Sharpe LT, Wandell BA (2001) Abnormal retinotopic representations in human visual cortex revealed by fMRI. Acta Psychologica 107:229247.

Nettleship E (1909) On some herediatry diseases of the eye. Transactions of the Ophthalmological Societies of the United Kingdom 29:57-198.

Neveu MM, von dem Hagen E, Morland AB, Jeffery G (2008) The fovea regulates symmetrical development of the visual cortex. Journal of Comparative Neurology 506:791-800.

O'Donnell FE, Jr., King RA, Green WR, Witkop CJ, Jr. (1978) Autosomal recessively inherited ocular albinism. A new form of ocular albinism affecting females as severely as males. Archives of Ophthalmology 96:1621-1625.

Popovic Z, Sjöstrand J (2001) Resolution, separation of retinal ganglion cells, and cortical magnification in humans. Vision Research 41:1313-1319. 
948

949

950

951

952

953

954

955

956

957

958

959

960

Puckett AM, DeYoe EA (2015) The attentional field revealed by single-voxel modeling of fMRI time courses. Journal of Neuroscience 35:5030-5042.

Qiu A, Rosenau BJ, Greenberg AS, Hurdal MK, Barta P, Yantis S, Miller MI (2006) Estimating linear cortical magnification in human primary visual cortex via dynamic programming. Neurolmage 31:125-138.

Reitsma DC, Mathis J, Ulmer JL, Mueller W, Maciejewski MJ, DeYoe EA (2013) Atypical retinotopic organization of visual cortex in patients with central brain damage: Congenital and adult onset. Journal of Neuroscience 33:13010-13024.

Saad ZS, DeYoe EA, Ropella KM (2003) Estimation of fMRI response delays. Neuroimage 18:494-504.

Schmitz B, Käsmann-Kellner B, Schäfer T, Krick CM, Grön G, Backens M, Reith W (2004) Monocular visual activation patterns in albinism as revealed by functional magnetic resonance imaging. Human Brain Mapping 23:40-52.

Schneider CA, Rasband WS, Eliceiri KW (2012) NIH image to ImageJ: 25 years of image analysis. Nature Methods 9:671-675.

Sereno MI, Dale AM, Reppas JB, Kwong KK, Belliveau JW, Brady TJ, Rosen BR, Tootell RB (1995) Borders of multiple visual areas in humans revealed by functional magnetic resonance imaging. Science 268:889-893.

Steinman RM (1965) Effect of target size, luminance, and color on monocular fixation. Journal of the Optical Society of America 55:1158-1165.

Summers CG (1996) Vision in albinism. Transactions of the American Ophthalmological Society 94:1095-1155.

Thomas MG, Kumar A, Mohammad S, Proudlock FA, Engle EC, Andrews C, Chan WM, Thomas S, Gottlob I (2011) Structural grading of foveal hypoplasia using spectraldomain optical coherence tomography: A predictor of visual acuity? Ophthalmology 118:1653-1660.

von dem Hagen EA, Hoffmann MB, Morland AB (2008) Identifying human albinism: A comparison of VEP and fMRI. Investigative Ophthalmology \& Visual Science 49:238249.

von dem Hagen EA, Houston GC, Hoffmann MB, Morland AB (2007) Pigmentation predicts the shift in the line of decussation in humans with albinism. European Journal of Neuroscience 25:503-511. 
980 von dem Hagen EA, Houston GC, Hoffmann MB, Jeffery G, Morland AB (2005) Retinal abnormalities in human albinism translate into a reduction of grey matter in the occipital cortex. European Journal of Neuroscience 22:2475-2480.

Wässle H, Grünert U, Röhrenbeck J, Boycott BB (1990) Retinal ganglion cell density and cortical magnification factor in the primate. Vision Research 30:1897-1911.

Wilk MA, Wilk BM, Langlo CS, Cooper RF, Carroll J (2017) Evaluating outer segment length as a surrogate measure of peak foveal cone density. Vision Research 130:57-66.

Wilk MA, McAllister JT, Cooper RF, Dubis AM, Patitucci TN, Summerfelt P, Anderson JL, Stepien KE, Costakos DM, Connor TB, Jr., Wirostko WJ, Chiang PW, Dubra A, Curcio CA, Brilliant MH, Summers CG, Carroll J (2014) Relationship between foveal cone specialization and pit morphology in albinism. Investigative Ophthalmology \& Visual

992 Wilson HR, Mets MB, Nagy SE, Kressel AB (1988) Albino spatial vision as an instance of arrested visual development. Vision Research 28:979-990. 